\title{
Estimating the extent of degradation of ruminant feeds from a description of their gas production profiles observed in vitro: comparison of models
}

\author{
M. S. Dhanoa ${ }^{1}$, S. Lopez ${ }^{2}$, J. Dijkstra ${ }^{3}$, D. R. Davies ${ }^{1}$, R. Sanderson ${ }^{1}$, B. A. Williams ${ }^{3}$, Z. Sileshi ${ }^{4}$ and \\ J. France ${ }^{5 *}$ \\ ${ }^{1}$ Institute of Grassland and Environmental Research, Plas Gogerddan, Aberystwyth SY23 3EB, UK \\ ${ }^{2}$ Department of Animal Production, University of Leon, 24007 Leon, Spain \\ ${ }^{3}$ Animal Nutrition Group, Wageningen Institute of Animal Sciences, Wageningen Agricultural University, \\ Marijkeweg 40, 6709 PG Wageningen, The Netherlands \\ ${ }^{4}$ Institute of Agricultural Research, PO Box 2003, Ethiopia \\ ${ }^{5}$ The University of Reading, Department of Agriculture, PO Box 236, Earley Gate, Reading RG6 6AT, UK
}

(Received 9 June 1998 - Revised 24 May 1999 - Accepted 19 July 1999)

\begin{abstract}
An evaluation of general models that describe gas production profiles is presented. The models are derived from first principles by considering a simple three-pool scheme and permit the extent of ruminal degradation to be calculated, as described in the companion paper. The models evaluated were the generalized Mitscherlich, simple Mitscherlich, generalized MichaelisMenten, simple Michaelis-Menten, Gompertz, and logistic. Five sets of gas production data consisting of 216 curves, obtained using a wide range of feeds (including straw, hay, silage, grain and various byproducts), were analysed to study the performance of these gas production models. Application of the non-sigmoidal models (simple Mitscherlich and Michaelis-Menten) to the data resulted in convergence problems and these models were found to be inadequate in many cases. Based on results of a pairwise comparison between models (variance ratio test), ranking of residual mean squares, lack-of-fit test, and of analyses of residuals, the generalized Mitscherlich and the generalized Michaelis-Menten models seemed particularly suited because of their flexibility to encompass sigmoidal and non-sigmoidal shapes of gas production profiles, whether symmetrical or not.
\end{abstract}

Rumen: Mathematical models: Gas production: Feed degradation

There is renewed interest amongst ruminant nutritionists in the use of gas production methods, where fermentation under controlled conditions allows the study of feed degradation. These in vitro methods involve incubating a feed sample in a buffered solution inoculated with rumen fluid, and measuring the resultant gas production periodically. This renewed interest is dictated by the inability of end-point quality measurement techniques, e.g. digestibility (Tilly \& Terry, 1963), to provide kinetic information. Such information is generally obtained using the in situ polyester bag method (Ørskov \& McDonald, 1979), though extensive use of animals and the fact that disappearance from the bag, rather than products of rumen fermentation, is measured tend to limit its utility. Availability of kinetic information from in vitro gas production curves of ruminant feeds has created a need for data analysis models in order to estimate nutritionally important variables. In the analysis of gas production data many mathematical functions have been adopted (Beuvink \& Kogut, 1993; Blümmel \& Ørskov, 1993; France et al. 1993; Schofield et al. 1994; Groot et al. 1996). The diversity of gas production profile shapes, whether an inflexion point exists or not, and, crucially, the relationship between the gas production kinetics and the extent of degradation in the rumen, requires the application of an appropriate model to describe the curves and to link the profile to ruminal degradation. Classical (sigmoidal) growth functions, in standard or alternative forms, have been used by some workers (Beuvink \& Kogut, 1993; Schofield et al. 1994). France et al. (1993) proposed a new function which is capable of modelling sigmoidal and nonsigmoidal shapes encountered in gas production studies, and showed a way to link the gas production kinetics in vitro to

\footnotetext{
Abbreviations: E, extent of ruminal degradation; EXP, exponential; GEXP, generalized Mitscherlich; GMM, generalized Michaelis-Menten; GOMP, Gompertz; LOG, logistic; MM, Michaelis-Menten; OM, organic matter; RMS, residual mean squares; rMSPE, root mean square prediction error; $\mathrm{S}_{0}$, potentially degradable fraction of the feed; VFA, volatile fatty acids; Y, yield factor relating gas produced to substrate degraded.
}

* Corresponding author: Professor J. France, fax +44 (0)118 935 2421, email j.france@ reading.ac.uk 
extent of degradation in the rumen. This link-up between in vitro gas production variables and substrate degradation in the rumen has now been extended further to include the use of classical functions by providing compartmental interpretations of these functions, as detailed in the companion paper (France et al. 2000). Knowing that microbial protein supply and production of volatile fatty acids (VFA) in the rumen are related to the extent of degradation, such a linkup is of high significance.

The main objective of the present paper was to evaluate four general functions (plus two special cases) which describe gas production profiles, by fitting them to the gas production curves obtained using a diverse range of ruminant feeds. The functions evaluated were the generalized Mitscherlich, generalized Michaelis-Menten, and growth functions, Gompertz and logistic. These functions were derived from first principles by considering a simple three-pool scheme, as described by France et al. (2000). The emphasis is on the behaviour of these functions under general application, not on in-depth mathematical or statistical properties.

\section{Materials and methods}

\section{Choice of models}

In this study, four models were selected for evaluation for the analysis of gas production data, i.e. generalized Mitscherlich $\left(\mathrm{GEXP}_{\mathrm{L}}\right)$, generalized Michaelis-Menten $\left(\mathrm{GMM}_{\mathrm{L}}\right.$ or $\left.\mathrm{GMM}_{0}\right)$, Gompertz $\left(\mathrm{GOMP}_{\mathrm{L}}\right.$ or $\left.\mathrm{GOMP}_{0}\right)$ and logistic $\left(\mathrm{LOG}_{\mathrm{L}}\right.$ or $\left.\mathrm{LOG}_{0}\right)$. The subscripts $\mathrm{L}$ and 0 denote the presence or absence of a discrete lag time $\mathrm{T}(\mathrm{h})$. In the case of the generalized Mitscherlich model a discrete lag, albeit small, is needed to prevent a mathematical discontinuity at zero time (France et al. 2000). In addition, special cases, based on the generalized Mitscherlich model, i.e. exponential $\left(\mathrm{EXP}_{\mathrm{L}}\right)$, and based on the generalized MichaelisMenten model, i.e. the Michaelis-Menten commonly used in enzyme kinetics $\left(\mathrm{MM}_{\mathrm{L}}\right.$ and $\left.\mathrm{MM}_{0}\right)$, were evaluated. From a number of functions that may describe gas production curves, these four models and their special cases can be derived from first principles by considering a simple compartmental scheme, as described by France et al. (2000). In that derivation, the gas technique is linked to animal production by constructing and evaluating substrate-based models that facilitate the calculation of extent of degradation in the rumen based on the kinetics of gas production. To derive the extent of degradation, a fractional passage rate has to be assumed, consistent with the approach in the in situ method that is currently used to estimate degradation of feed components in the rumen as applied in feed evaluation systems (Agricultural and Food Research Council, 1992; Tamminga et al. 1994).

\section{The data}

A sample set of 216 gas production profiles was obtained for evaluation of the previously mentioned functions. The total sample set could be separated into five subsets. Certain characteristics of subset 5 differed from those of the other subsets (explained later, p. 134). Therefore, subsets 1-4 are referred to as the main set. The main set included concentrates, grass, silage, hay, straw, and pure substrates (cellulose) to represent the diversity of the shapes of the gas production profiles. The samples represented both tropical and temperate climates, and the laboratories where fermentation studies were carried out were situated in Ethiopia (forty-nine profiles), the Netherlands (thirty-nine profiles), Spain (twelve profiles) and UK (sixteen profiles).

The Ethiopian set (subset 1) comprised (a) five cuts of each of three cultivars (ILCA 14983, 14984, X) of Napier grass (Pennisetum purpureum Schumach) and one cultivar of pigeon grass (Heteropogon white), (b) four cuts of leaf and 'branch' of tree lucerne (Chamaecytisus palmensis), (c) pooled samples from three replicate plots at each of six harvests of reed canary grass (Phalaris arudinacea L.), and (d) fifteen crop residue-based forages comprising whole stover and fractions (leaf, leaf sheath, stem) of two cultivars of sorghum (Dinkimarsh, X35/24) and fractions (leaf, stem, panicle, chaff) of tef grass (Eragrostica abyssinica), whole wheat straw and whole barley straw. For more details see Sileshi et al. (1996).

The Dutch set (subset 2) consisted of (a) untreated and ammoniated wheat (Triticum aestivum) straw and maize (Zea mays) silage (Williams et al. 1995), (b) lupin (Lupinus angustifolius), pea (Pisum sativum) and rapeseed (Brassica napus) from a feed company, (c) maize, soyabean (Glycine max) hulls, soyabean meal and wheat from a commercial source, (d) sorghum (Sorghum bicolor) straw, (e) red clover (Trifolium pratense), white clover (T. repens) and ryegrass (Lolium perenne) received from the Louis Bolk Institute (Driebergen, The Netherlands), (f) barley (Hordeum vulgare), cellulose, good hay, poor hay (both Lolium perenne) and soyabean meal samples as used in a ring test and provided by ADAS (UK) and (g) samples of rice (Oryza sativa) straw from ten varieties as fed to animals and also samples of their refusals (Williams et al. 1996).

The Spanish hays (subset 3) were from sown pastures (six hays) and the remainder from permanent mountain meadows growing at various altitudes and irrigation regimes with a high diversity of botanical composition (Lopez et al. 1998). The six sown pasture hays consisted of white clover, lucerne (Medicago sativa), mixture of vetch (Vicia sativa) and oat (Avena sativa) cereal, red clover, grass-clover mixture and mixed grass sward. Four of the mountain meadow hays were from a June cut and the other two from a regrowth cut in September.

The UK set (subset 4) was composed of (a) different particle sizes as degraded by fungi (France et al. 1993), (b) same samples as in (a) but degraded by bacteria, (c) one fresh grass sample and one $106 \mathrm{~d}$ grass silage sample and (d) one most fermentable, one least fermentable sample of Gliricidia sepium provenance and one Gliricidia maculata sample (France et al. 1993). Further details of incubation procedures are described by Theodorou et al. (1994).

For visual appreciation of the diversity of shapes, all these profiles are shown in Fig. 1(a) as a simple plot of timespecific cumulative gas production data. Some differences between profiles are due to the different amounts of sample used for the in vitro incubations (ranging from approximately $0 \cdot 2$ to $1 \mathrm{~g} \mathrm{DM}$ ), to the different incubation times used in each laboratory (the final time ranged between 96 and $192 \mathrm{~h}$ ), and to rumen fluid obtained from different donor 


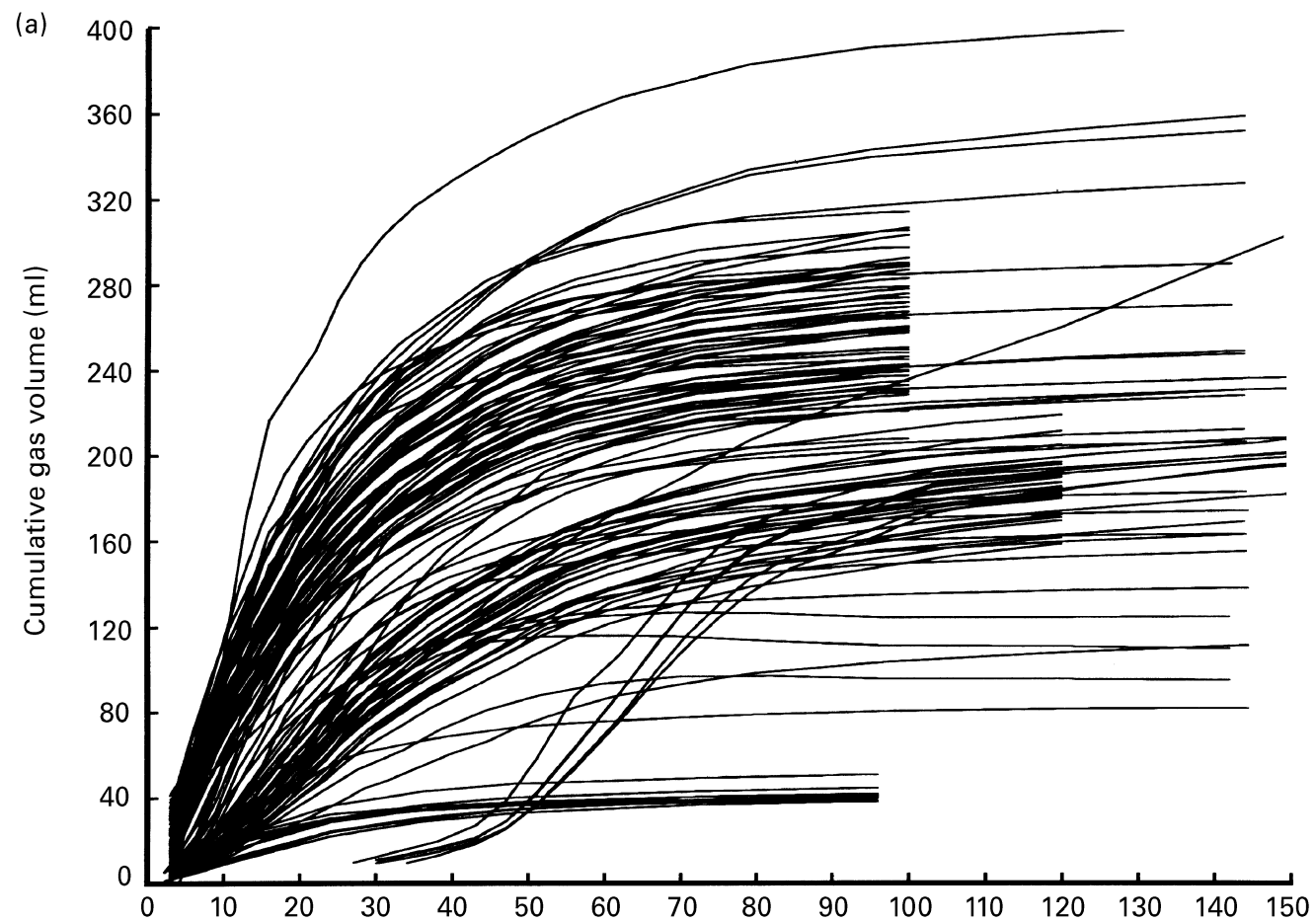

Time since inoculation (h)

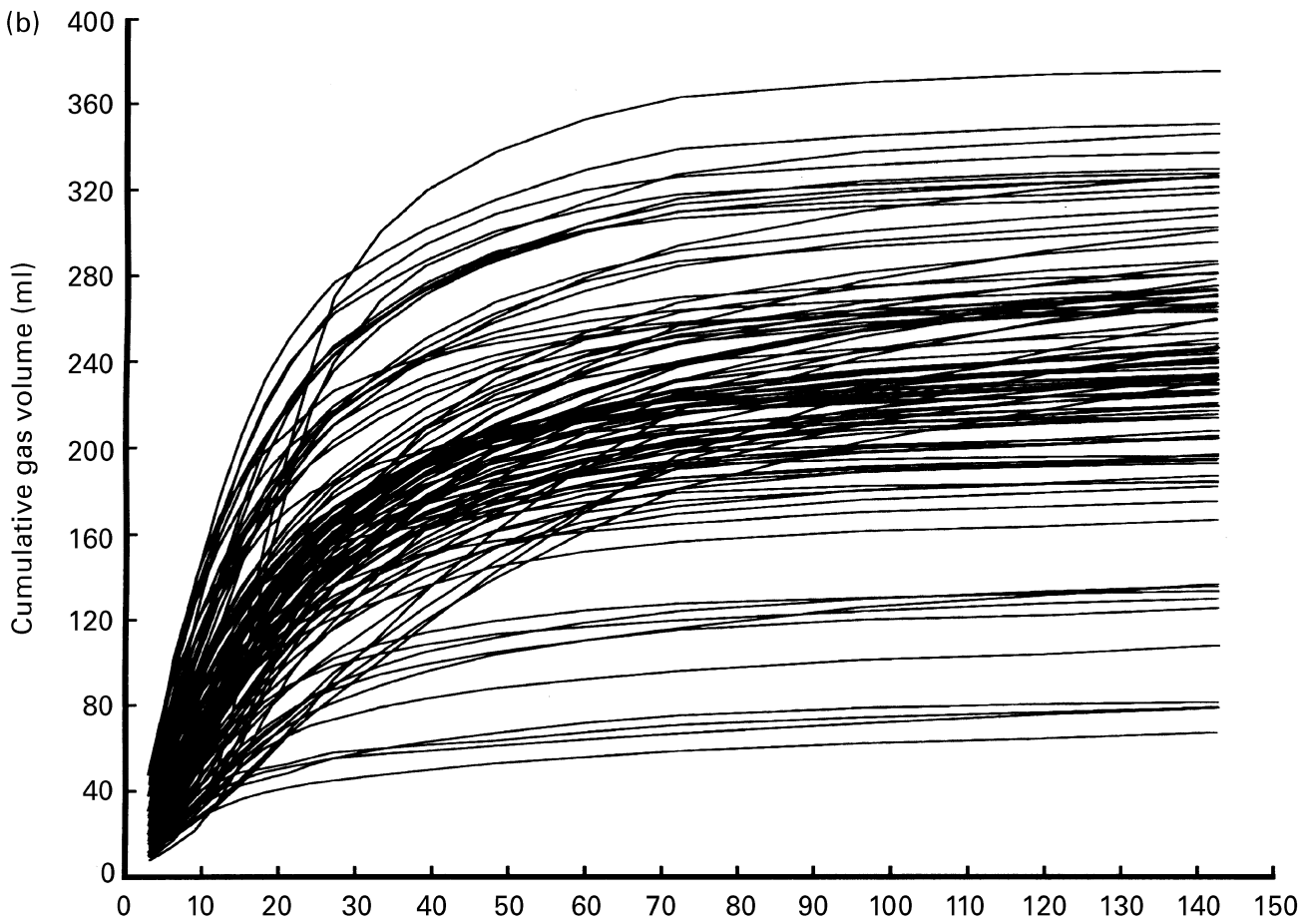

Time since inoculation (h)

Fig. 1. Simple plot of gas production profiles used in the present study. (a) Main set comprising 116 profiles; (b) subset 5 comprising 100 profiles.

animals and fed on various diets. The different experimental procedures used for each case (use of manual or automatic systems, different buffers and media, etc.) may also be reflected in some of the differences between profiles.
Finally, as the gas production technique is based on pressure measurements, the variation in location of the laboratories, related to differences in altitude and climate, may also result in differences in the recorded profiles. 
Subset 5 consisted of fifty Mediterranean animal feeds. This included concentrates, whole-crop legumes, cereal grasses, permanent meadow hays, cereal and legume straws and byproducts from the fruits and vegetables processing industry, such as ensiled sugarbeet (Beta vulgaris) pulp, dried artichoke (Cynara scolymus) leaves and broccoli (Brassica cleracea) plant hay, all collected in Spain. Two separate gas production runs (final incubation time $144 \mathrm{~h}$ ) were made for each of the feeds to give a total of 100 profiles. In contrast to the main set, duplicate bottles were used in each run and gas production data recorded for each of the two bottles were assigned to the same profile (France et al. 1998). This replication within a run allows for specific statistical assessment (lack-of-fit test) to be made that cannot be performed for the data in the main-set. All the profiles in the subset 5 are shown in Fig. 1(b). In this dataset, differences among profiles are only due to the different substrates fermented, as all of them were incubated under the same experimental conditions.

\section{Computational analysis}

The selected models were fitted to all the previously mentioned data sets comprising 216 gas production profiles. Non-linear curve fitting was done in MLP (Maximum Likelihood Program; Ross, 1987) and SAS (version 6, 1989; Statistical Analysis Systems Institute Inc., Cary, NC, USA), which are particularly suited for handling and processing large data sets. The initial values were inferred from the original papers and the curves were fitted by nonlinear regression methods using a maximum of fifty iterations. As these models are apparently suitable for use by the scientists at large, no attempt was made to finetune individual curve fits. Thus, all models were used on the same data sets and same conditions generating 2376 (216 curves $\times 11$ models) full sets of solutions. All the models were fitted in their non-linear form. However, the GOMP and LOG models contain some expressions which involve several parameters that can be considered as single linear scaling constants. Therefore, fitting of the GOMP model was facilitated taking $\mathrm{b} / \mathrm{c}$ as a single parameter $\alpha$, and fitting of the LOG model by taking bK as a single parameter $\alpha$ and $\mathrm{S}_{0} /\left(\mathrm{K}-\mathrm{S}_{0}\right)$ as a single parameter $\beta$ (see France et al. 2000, for original form).

In order to determine the ruminal extent of degradation from the profile of gas production, it is necessary to know the quantity of the potential degradable fraction of the feed $\left(\mathrm{S}_{0}, \mathrm{~g} \mathrm{DM}\right)$. Thus, the residue remaining at final incubation time and retained in crucibles after filtering and washing with distilled water was obtained for most samples used in this study, and $\mathrm{S}_{0}$ calculated by subtracting this residue from the quantity of feed component originally incubated. Since $\mathrm{S}_{0}$ and $\mathrm{Y}$ (the yield factor relating gas produced to substrate degraded; ml gas/g degradable DM) are only linear scale parameters, their product $\left(\mathrm{YS}_{0}\right)$ was fitted as a single parameter A (asymptotic gas production; $\mathrm{ml}$ gas). $\mathrm{S}_{0}$ is a known quantity and thus the value of $\mathrm{Y}$ can easily be calculated after the fit by dividing $\mathrm{A}$ by $\mathrm{S}_{0}$. The extent of degradation in the rumen for each model is calculated using the equations derived by France et al. (2000), assuming a fractional passage rate of $0 \cdot 02 / \mathrm{h}$. The statistical package GENSTAT
(Genstat 5 Committee, 1987) was used for numerical integration of the non-analytical integral in these equations, applying the AREA function based on cubic interpolation. The non-analytical integral for the GEXP model is sensitive to the time-interval used in the numerical integration, because of the presence of $\sqrt{ } \mathrm{t}$ in the denominator. Therefore the use of the slightly different form of this non-analytical integral as presented by France et al. (1993) is recommended. Thus, the extent of degradation for each model is calculated using the estimates of the specific parameters in each model, the measured value of the potentially degradable fraction, and the assumed value of the fractional passage rate.

To evaluate the performance of the models relative to each other, several criteria were utilized. These included the convergence success rate, the ranking of residual mean squares (RMS) from the fit of each model, the lack-of-fit test, the $F$ test or variance ratio test, and various tests based on the pattern of distribution of residuals. All regression analyses and graphics were done using GENSTAT (Genstat 5 Committee, 1987).

\section{Results \\ Fitting behaviour}

For each of the eleven models applied in this study the nonconvergence and unacceptable fit rates are presented in Table 1. Non-convergence can be symptomatic of an illconditioned (i.e. parameter values tending to 0 or to biologically unacceptable values, or high correlation amongst parameters) or an inappropriate model. Fitting of the GOMP and LOG models was facilitated by taking linear parameters as a single parameter (see above). The GEXP model and the $\mathrm{GMM}_{0}$ model resulted in smallest convergence failures $(0.9 \%$ or two out of 216 profiles). The convergence failure rate exceeded $5 \%$ for the $\mathrm{EXP}_{0}, \mathrm{LOG}_{\mathrm{L}}, \mathrm{GMM}_{\mathrm{L}}, \mathrm{MML}_{\mathrm{L}}$ and $\mathrm{MM}_{0}$ models.

Since lag and substrate fractional degradation rate cannot be negative, several conditions on model parameters have to

Table 1. Non-convergence and unacceptable fit rates (\% of total) for the models: generalized Mitscherlich with lag (GEXP $\left.\mathrm{L}_{\mathrm{L}}\right)$, exponential with lag $\left(E_{X} P_{L}\right)$ and without lag $\left(E_{X P_{0}}\right)$, Gompertz with lag $\left(G O M P_{L}\right)$ and without lag $\left(\mathrm{GOMP}_{0}\right)$, logistic with lag $\left(\mathrm{LOG}_{\mathrm{L}}\right)$ and without lag $\left(\mathrm{LOG}_{0}\right)$, generalized Michaelis-Menten with lag $\left(\mathrm{GMM}_{\mathrm{L}}\right)$ and without lag $\left(\mathrm{GMM}_{0}\right)$ and Michaelis-Menten with lag $\left(\mathrm{MM}_{\mathrm{L}}\right)$ and without lag $\left(\mathrm{MM}_{0}\right)$ fitted to the whole data set (total number of profiles is 216 )

\begin{tabular}{|c|c|c|}
\hline Model & Non-convergence & Unacceptable fit \\
\hline GEXP & 0.9 & $1.4^{*}$ \\
\hline$E X P_{L}$ & 4.6 & $11 \cdot 6^{*}$ \\
\hline $\mathrm{EXP}_{0}$ & $9 \cdot 7$ & 0 \\
\hline $\mathrm{GOMP}_{\mathrm{L}}$ & $4 \cdot 2$ & $9.7^{*}$ \\
\hline $\mathrm{GOMP}_{0}$ & 3.7 & 0 \\
\hline $\mathrm{LOG}_{\mathrm{L}}$ & 6.5 & $32 \cdot 4 \dagger\left(+20 \cdot 8^{*}\right)$ \\
\hline $\mathrm{LOG}_{0}$ & $3 \cdot 2$ & $19.9 \dagger$ \\
\hline $\mathrm{GMM}_{\mathrm{L}}$ & $17 \cdot 1$ & $68 \cdot 1^{*}$ \\
\hline $\mathrm{GMM}_{0}$ & 0.9 & 0 \\
\hline $\mathrm{MM}_{\mathrm{L}}$ & $5 \cdot 1$ & $1.9^{*}$ \\
\hline $\mathrm{MM}_{0}$ & $6 \cdot 0$ & 0 \\
\hline
\end{tabular}

* Negative estimates of the lag parameter $T(h)$.

$\dagger$ Negative estimates of $S_{0} /\left(K-S_{0}\right)$ i.e. $K<S_{0}$, where $S_{0}$ is the potentially degradable fraction of the feed. 
be satisfied (France et al. 2000). In the case of the EXP, GOMP, LOG and GMM models, the introduction of a discrete lag parameter resulted in $10 \%$ or more unacceeptable solutions (negative estimate of lag parameter; Table 1). A discrete lag parameter less than $0 \mathrm{~h}$ implies a positive intercept at zero time, which for gas production is inadmissible. However, when the lag parameter was included in the model but constrained to be non-negative, convergence failures increased considerably (results not shown). The inclusion of the lag parameter allows the non-sigmoidal models to mimic approximate sigmoidal behaviour. Without the lag parameter, the non-sigmoidal models cannot fit sigmoidal profiles satisfactorily and convergence problems or high residual sums of squares occur. Growth functions in general, but the GMM model in particular, 'used' the lag parameter as a shift parameter on the time-axis in preference to changes in the shape parameter in order to describe gas production profiles with rapid gas production in the earlier stages. Omitting the lag-parameter for these models resolved this problem. A non-zero lag is necessary to fit the GEXP model to prevent a mathematical discontinuity at zero time (France et al. 2000). For this particular model, any problems in fitting the lag parameter with negative values may be overcome by fixing the lag parameter to a point at which the fitted curve assumes its minimum, as described by France et al. (1993). In addition to problems with the lag parameter, a number of unacceptable solutions were obtained in case of the LOG model. In the LOG model, the constraint $\mathrm{K} \geqslant \mathrm{S}_{0}$ has to be satisfied (see France et al. 2000); solutions with $\mathrm{K}<\mathrm{S}_{0}$ are unacceptable. When forcing $\mathrm{K}$ to be larger than $\mathrm{S}_{0}$, the value $\mathrm{S}_{0} /\left(\mathrm{K}-\mathrm{S}_{0}\right)$ tends to zero, thus evolving to the $\mathrm{EXP}_{\mathrm{L}}$ model.

Based on the results of non-convergence and unacceptable fit rates, four sigmoidal models (i.e. $\mathrm{GEXP}_{\mathrm{L}}, \mathrm{GOMP}_{0}$, $\mathrm{LOG}_{0}$, and $\mathrm{GMM}_{0}$ ), together with two non-sigmoidal submodels $\left(\mathrm{EXP}_{\mathrm{L}}\right.$ and $\left.\mathrm{MM}_{\mathrm{L}}\right)$ were identified as more viable than the other models, and therefore further evaluation was done for these six models only.

\section{Model performance}

Table 2 shows the results of a pairwise comparison between models using an $F$ test or variance ratio test (Motulsky \& Ransnas, 1987). For comparisons in which the GEXP is one of the models of a pair, the partial $F$ test rather than the variance ratio was calculated, because models differ by one extra parameter. The GEXP and GMM were superior to the standard growth functions (GOMP and LOG) and to the non-sigmoidal functions (MM and EXP). For the main set, the GEXP was better $(P<0.05)$ in describing $84 \%$ of the profiles and never significantly worse than the GMM, but for subset 5, the GEXP was better in $26 \%$ of the profiles but worse in $41 \%$ of the profiles. The ranking of RMS from the fit of each model within a profile and averaged over the whole data set gave mean scores of 1.75, 3.37, 2.29, 4.93, 4.88 and 3.78 for $\operatorname{GEXP}_{\mathrm{L}}, \mathrm{EXP}_{\mathrm{L}}, \mathrm{GMM}_{0}, \mathrm{MM}_{\mathrm{L}}, \mathrm{GOMP}_{0}$ and $\mathrm{LOG}_{0}$ respectively. The percentage cases with minimum RMS were $47,3,44,1,1$ and 4 , whilst cases with maximum RMS were $0,9,0,56,35$ and 0 respectively. The percentage of profiles in subset 5 in which the variance attributed to lack-of-fit significantly exceeds the pure error
Table 2. Pairwise comparison between models (GEXP $P_{L}$, generalized Mitscherlich with lag; $\mathrm{EXP}_{\llcorner}$, exponential with lag; $\mathrm{GMM}_{0}$, generalized Michaelis-Menten without lag; $M_{\mathrm{L}}$, Michaelis-Menten with lag; $\mathrm{GOMP}_{0}$, Gompertz without lag; $\mathrm{LOG}_{0}$, logistic without lag) using either a partial $F$ test or variance ratio test, showing the percentage of cases where the model specified in the row was significantly

$(P<0.05)$ superior to the model specified in the column

\begin{tabular}{|c|c|c|c|c|c|c|}
\hline & $\mathrm{GEXP}_{\mathrm{L}}$ & $\mathrm{EXP}_{\mathrm{L}}$ & $\mathrm{GMM}_{0}$ & $\mathrm{MM}_{\mathrm{L}}$ & $\mathrm{GOMP}_{0}$ & $\mathrm{LOG}_{0}$ \\
\hline \multicolumn{7}{|c|}{ Main set $(n 116)$} \\
\hline GEXP & & $84^{*}$ & $58^{*}$ & $95^{\star}$ & $90^{*}$ & $91^{*}$ \\
\hline $\mathrm{EXP}_{L}$ & $0^{*}$ & & 29 & 54 & 38 & 33 \\
\hline $\mathrm{GMM}_{0}$ & $0^{*}$ & 41 & & 73 & 60 & 53 \\
\hline $\mathrm{MM}_{\mathrm{L}}$ & $0^{*}$ & 1 & 3 & & 15 & 12 \\
\hline $\mathrm{GOMP}_{0}$ & $0^{*}$ & 13 & 12 & 31 & & 7 \\
\hline $\mathrm{LOG}_{0}$ & $0^{*}$ & 26 & 15 & 48 & 8 & \\
\hline \multicolumn{7}{|c|}{ Subset 5 (n 100) } \\
\hline GEXPL & & $48^{*}$ & $26^{*}$ & $86^{*}$ & $70^{*}$ & $46^{*}$ \\
\hline $\mathrm{EXP}_{\mathrm{L}}$ & $0^{*}$ & & 7 & 55 & 50 & 1 \\
\hline $\mathrm{GMM}_{0}$ & $41^{*}$ & 65 & & 81 & 79 & 67 \\
\hline $\mathrm{MM}_{\mathrm{L}}$ & $5^{*}$ & 26 & 0 & & 30 & 17 \\
\hline $\mathrm{GOMP}_{0}$ & $1^{*}$ & 6 & 7 & 45 & & 1 \\
\hline $\mathrm{LOG}_{0}$ & $2^{*}$ & 9 & 12 & 57 & 31 & \\
\hline
\end{tabular}

* Partial $F$ test was used because models differed by one extra parameter; the variance ratio test was used for all other pairs of models.

variance was evaluated using the lack-of-fit test (Draper $\&$ Smith, 1981). This test could not be performed for the main set due to lack of replication from which estimates of pure error are derived; in subset 5, bottle replicates allowed the calculation of pure error estimates. The percentage of profiles with significant lack-of-fit $(P<0.05)$ were: $\mathrm{GEXP}_{\mathrm{L}}$, 25; EXP $\mathrm{L}, 37 ; \mathrm{GMM}_{0}, 21 ; \mathrm{MM}_{\mathrm{L}}, 52 ; \mathrm{GOMP}_{0}, 49 ; \mathrm{LOG}_{0}$, 37.

The root mean square prediction error (rMSPE; Bibby \& Toutenberg, 1977) is an indicator of overall deviation between observed and fitted values. The rMSPE was calculated for each valid fit as:

$$
\operatorname{rMSPE}=\sqrt{ }\left\{\left[\Sigma(\mathrm{y}-\hat{\mathrm{y}})^{2}\right] / n\right\}
$$

where $\mathrm{y}$ and $\hat{\mathrm{y}}$ denote observed and fitted values of gas volume respectively, and $n$ the number of data points defining each individual curve. In Fig. 2, the distributions of rMSPE of four models (GEXP, GMM, GOMP and LOG) are presented using box-and-whisker plots (Tukey, 1977). The distribution of rMSPE of the models, in particular the GEXP, is skewed towards smaller values. The solutions with outliers were not rejected because large rMSPE was due to a few ill-fitting observations at the start of the profiles. Rapidly fermenting fractions of substrate may be implicated in generating these non-typical data values. The results of the variance ratio or $F$ test, the lack-of-fit test, and the rMSPE suggest that the GEXP and the GMM are superior models, and the GOMP and MM are inferior models.

\section{Analyses of residuals}

Time-related patterns of residuals after the fit of the models were analysed. For all the models in this study, the firstorder serial or autocorrelation of the residuals varied between 0.75 and 0.95 , indicating significant cyclic trends in a positively correlated series of residuals. The residual 


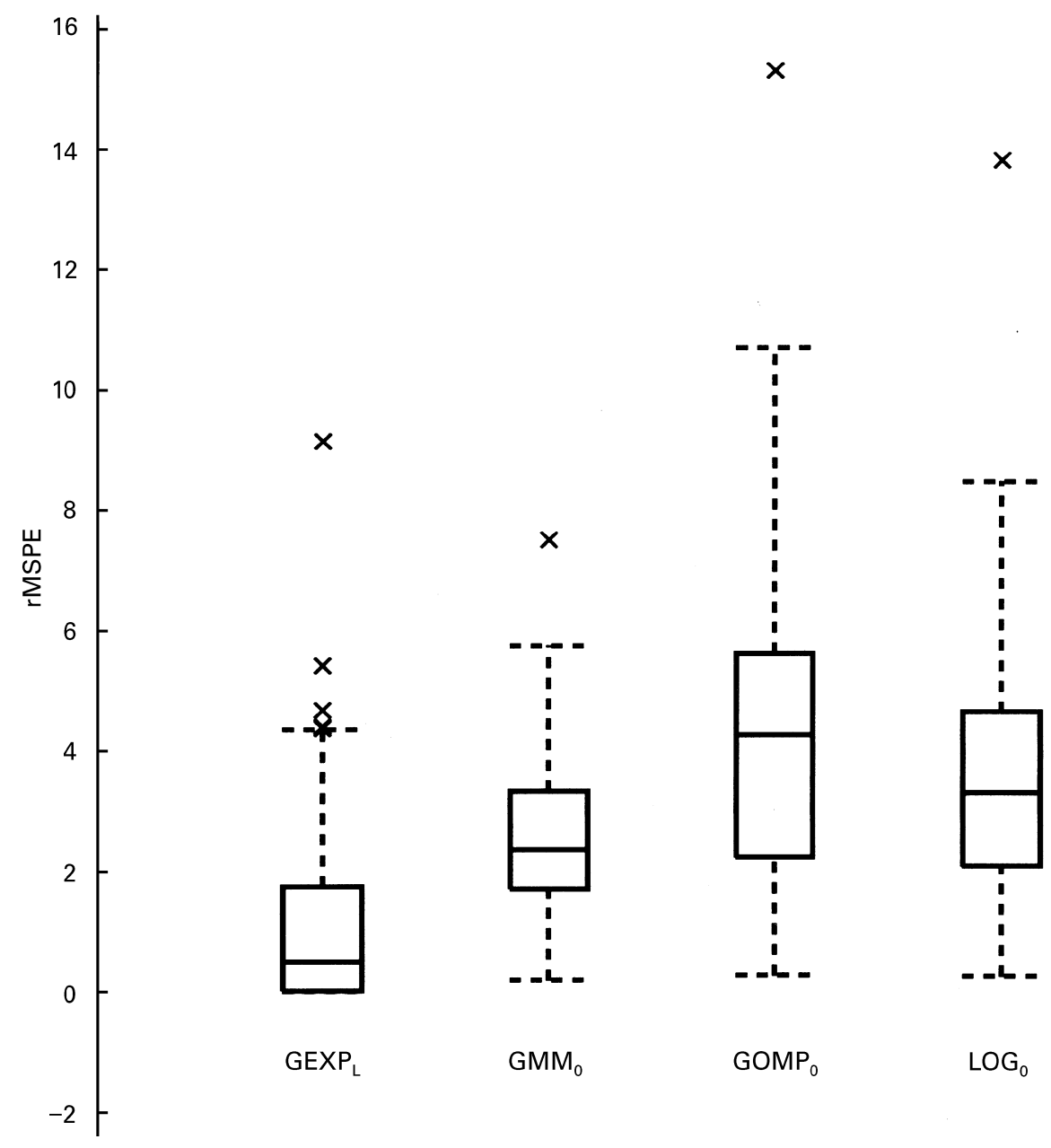

Fig. 2. Box-and-whisker plots (Tukey, 1977) of the distribution of root mean square prediction error (rMSPE) from the generalized Mitscherlich with lag (GEXP $\mathrm{L}_{\mathrm{L}}$ ), generalized MichaelisMenten without lag $\left(\mathrm{GMM}_{0}\right)$, Gompertz without lag $\left(\mathrm{GOMP}_{0}\right)$ and logistic without lag $\left(\mathrm{LOG}_{0}\right)$ models. Outliers are labelled as ' $X$ ' and the median of the distribution splits the box into two parts. Unequal partitioning of the box indicates skewed distribution.

behaviour of the models can be clearly seen from the results of calculating the number of runs of sign of the residuals, i.e. counting the number of sequences of the same sign (Table 3). A low number of runs from fitting a model indicates systematic under- or over-fitting, whereas a

Table 3. Percentage of curves expressed according to number of runs of sign of the residuals from fitting the generalized Mitscherlich with lag $\left(G E X P_{L}\right)$, exponential with lag $\left(E_{X} P_{L}\right)$, generalized MichaelisMenten without lag $\left(\mathrm{GMM}_{0}\right)$, Michaelis-Menten with lag $\left(\mathrm{MM}_{\mathrm{L}}\right)$, Gompertz without lag $(\mathrm{GOMP})$ and logistic without lag $\left(\mathrm{LOG}_{0}\right)$ models (total number of profiles is 216 )

\begin{tabular}{lccccc}
\hline & \multicolumn{5}{c}{ No. of runs of residual sign } \\
\cline { 2 - 6 } Model & $<4$ & $4-5$ & $6-7$ & $8-9$ & $>9$ \\
\hline GEXP $_{\mathrm{L}}$ & 13 & 57 & 18 & 10 & 2 \\
EXP $_{\mathrm{L}}$ & 24 & 67 & 7 & 2 & 0 \\
$\mathrm{GMM}_{0}$ & 15 & 56 & 25 & 3 & 1 \\
$\mathrm{MM}_{\mathrm{L}}$ & 39 & 55 & 6 & 0 & 0 \\
$\mathrm{GOM}_{0}$ & 30 & 62 & 7 & 1 & 0 \\
LOG $_{0}$ & 31 & 58 & 9 & 2 & 0 \\
\hline
\end{tabular}

larger number of runs, in combination with the positive autocorrelation noted before, indicates increased randomness of the residuals and ability of that model to describe the profiles more adequately. The GEXP and GMM models exhibited a higher number of runs of sign in the upper classes than did the other models (Table 3). Some $90 \%$ or more of the profiles fitted using EXP, MM, GOMP and LOG had five or less runs of residual sign and consequently a relatively high systematic bias. Most of the variation in residuals is at the early stages of incubation, with particularly the GOMP and LOG models showing a pattern of residuals (results not shown). The flexibility of the GEXP and GMM models apparently coped better in describing the profiles than the GOMP and LOG models.

\section{Ruminal extent of degradation}

The calculated ruminal extent of degradation for all models showed a large variation (between 4 and $73 \%$ ). A high correlation amongst estimates of extent of degradation from the models (in excess of 0.95 ) was calculated, indicating 
consistency apart from a possible mean bias. To illustrate such differences between the models, in Fig. 3(a)-(c) individually matched comparisons are presented. Fig. 3(a) demonstrates the negative mean bias of estimates from the $\mathrm{GMM}_{0}$ model in comparison with the GEXP $\mathrm{L}_{\mathrm{L}}$ model. The extent of degradation calculated using the $\mathrm{GOMP}_{0}$ model was similar to that using the $\mathrm{GEXP}_{\mathrm{L}}$ model, whereas the $\mathrm{LOG}_{0}$ model seemed to overestimate the extent of degradation in comparison with the $\mathrm{GEXP}_{\mathrm{L}}$ model (Fig. 3(b) and 3(c) respectively).

\section{Discussion}

\section{Adequacy of models}

The choice of a data analysis model depends on the underlying kinetics which may be determined theoretically, as in the case of mechanistic models, or empirically. Most published gas production models are empirical and fail to link the gas production to substrate disappearance. In the present analyses it was decided to construct mechanistic models of gas production based on the potentially degradable substrate and its fractional degradation rate (France et al. 2000). The models were evaluated using a wide range of gas production profiles (a total of 216 profiles), obtained using a range of substrates, to reflect wide variety in shape (non-sigmoidal and varying degree of sigmoidicity). It is visually apparent that subset 5 overall has less sigmoidal profiles and also smaller lag phase than the main set, and such differences might result in variations in relative model behaviour. Indeed, using a partial $F$ test or a variance ratio test (Motulsky \& Ransnas, 1987) pairwise comparison results of residual variances differ somewhat between the main set and subset 5 (Table 2).

Exponential (or monomolecular) functions have been used extensively in biological and physical sciences. In animal sciences, the well-known use of the exponential function is to model degradation of feeds when using the in situ Dacron bag technique (Ørskov \& McDonald, 1979). However, these first-order kinetics models are non-sigmoidal and have been found to be inadequate in many cases (Beuvink \& Kogut, 1993; France et al. 1993). Like the EXP model, the simple MM model is non-sigmoidal. Application of these non-sigmoidal models to the data in the present study, which are partly sigmoidal in nature, resulted in convergence problems (Table 1) and high RMS, and analyses of the residuals confirmed the inadequacy of these models to model gas production (Table 3 ).

The main inspiration for reparameterization of the LOG, GOMP and other classical sigmoidal growth functions by Zwietering et al. (1990) was to obtain a fitted parameter that directly gave maximum growth rate of microorganisms (dy/dt) which occurs at the point of inflexion. However, that requires the presence of an inflexion point, which in gas production studies is not always the case. All models which reach an asymptote (upper or lower) as time approaches infinity, possess a point where half the maximal response is obtained. Only in symmetrical functions will half-life and inflexion point coincide. Therefore, at least in gas production studies growth rate at the half-life time has wider range and application both for sigmoidal and non-sigmoidal shapes.

Application of sigmoidal models to the data in the present study showed differences between standard growth functions (GOMP and LOG) on the one hand and GEXP and GMM on the other hand. The latter two models are superior to the growth functions as shown by the results of the variance ratio test, lack-of-fit test, and analyses of residuals. The GOMP function is clearly unable to describe the whole range of shapes and may require subjective judgement to decide when to use it in preference to the non-sigmoidal curves. The behaviour of the LOG model was slightly better than that of the GOMP, as indicated by the results of the ranking of RMS and lack-of-fit tests. However, the unacceptable fit rates (related to the restriction of non-negative substrate degradation rate) of the LOG model were much higher (Table 1), which implies that the LOG model is not necessarily a proper alternative. Schofield et al. (1994) compared the EXP, LOG and GOMP models using two gas production profiles of cellulose fermentation. They did not observe consistent differences in fitting behaviour. Whilst this may be related to the specific profiles of the two substrates used in their study, the different forms of LOG and GOMP models in the study of Schofield et al. (1994) and in the present analysis may help to explain the lack of agreement. An initial gas volume (positive intercept at zero time) arises unavoidably with application of the LOG and GOMP as given by Schofield et al. (1994). These functions were originally derived to represent the growth of organisms, starting at some positive value at zero time. In our derivation of the GOMP and LOG models, presented in the companion paper (France et al. 2000), the predicted gas volume at the start of incubation is zero.

In contrast to the GOMP and LOG models, the GEXP and GMM models converged satisfactorily in almost all cases. Moreover, systematic bias of these two models was lower than that of the other functions. The fitting results using the large dataset in the present study do not indicate superior behaviour of either of the two models in comparison with each other. A partial $F$ test showed that the GEXP model was better than the GMM model in describing profiles in the main set, but with subset 5 the GMM model was better (Table 2). The main set and subset 5 differ from each other in samples included in the set as well as in incubation procedures, that may affect the profiles. The difference in performance of both models was almost negligible for ranking of RMS and the lack-of-fit test. Thus, both models seem equally suited to describe the profiles because of their flexibility to encompass sigmoidal and non-sigmoidal behaviour, whether symmetrical or not.

\section{Phasic behaviour}

As stated earlier the lag or zero response phase is a feature of in vitro gas production. In this period either no degradation occurs, or it occurs at a greatly reduced rate. Factors affecting the lag time include hydration of the substrate and bacterial attachment and colonization of insoluble substrate. Zero slope during lag phase is theoretically not consistent with models which tend to a lower asymptote (i.e. positive $y$ intercept at zero time) as is the case for the original forms of the Gompertz and logistic models (the growth function application). France et al. (1993) accommodated this aspect by assuming a discrete lag in their GEXP model. 
(a)

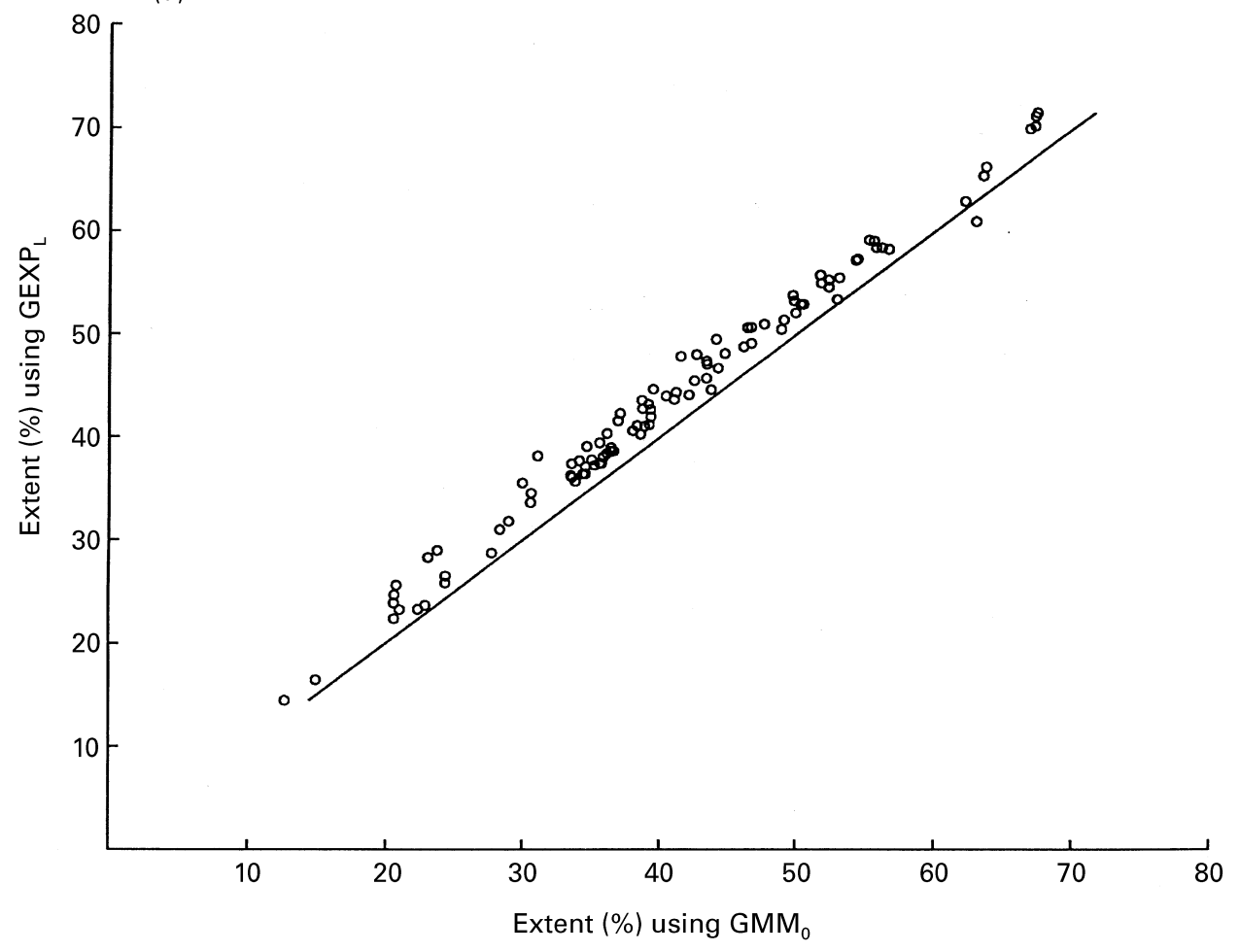

(b)

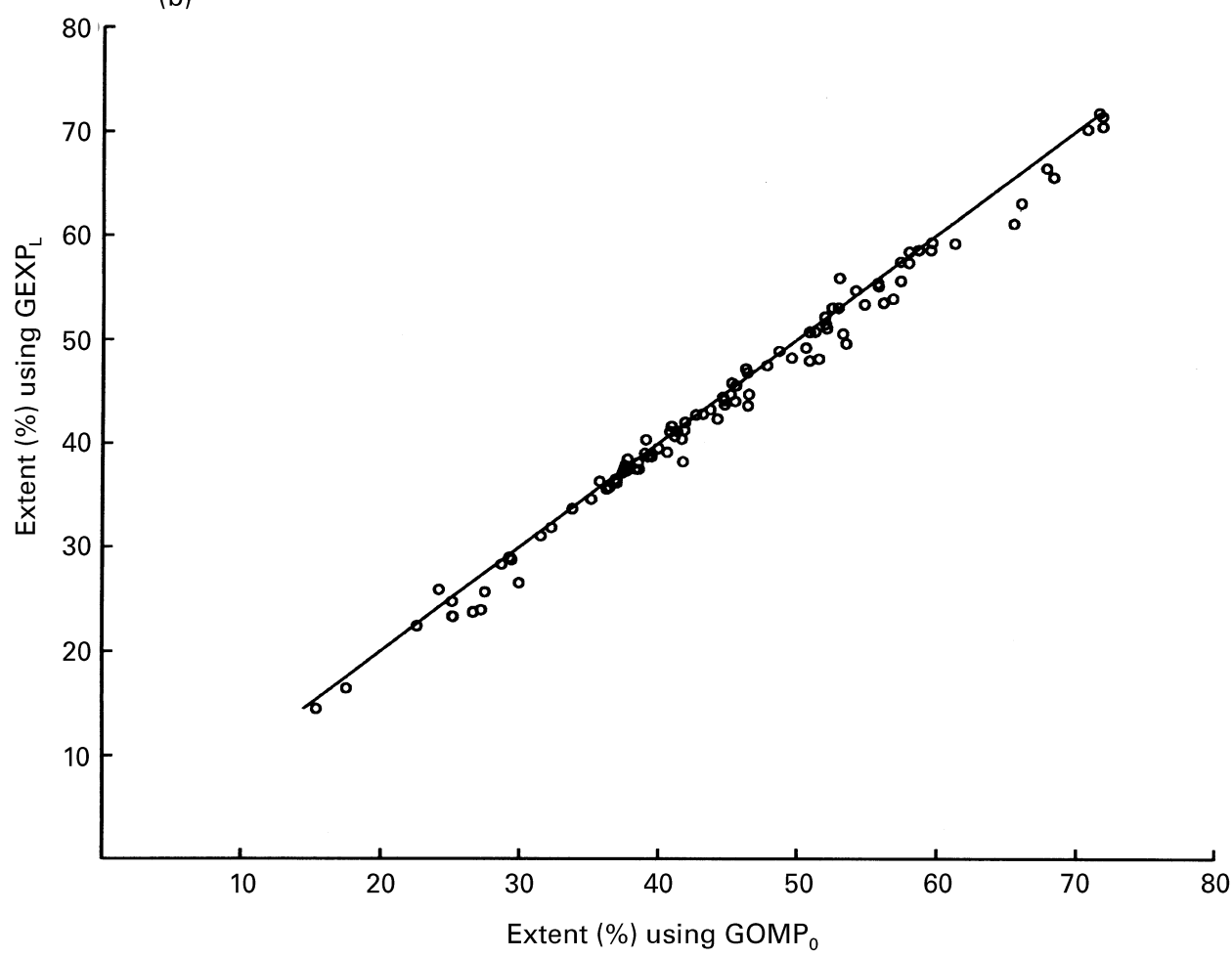

Fig. 3. Individually matched comparisons of calculated extent of degradation. (a) Generalized Mitscherlich with lag (GEXP $\mathrm{L}) v$. generalized Michaelis-Menten without lag $\left(G_{M M}\right)$, (b) GEXP $v_{L}$. Gompertz without lag $\left(G O M P_{0}\right)$, and (c) GEXP $v_{L}$. logistic without lag $\left(L_{0} O G_{0}\right)$ with reference to the line of equality. 
(c)

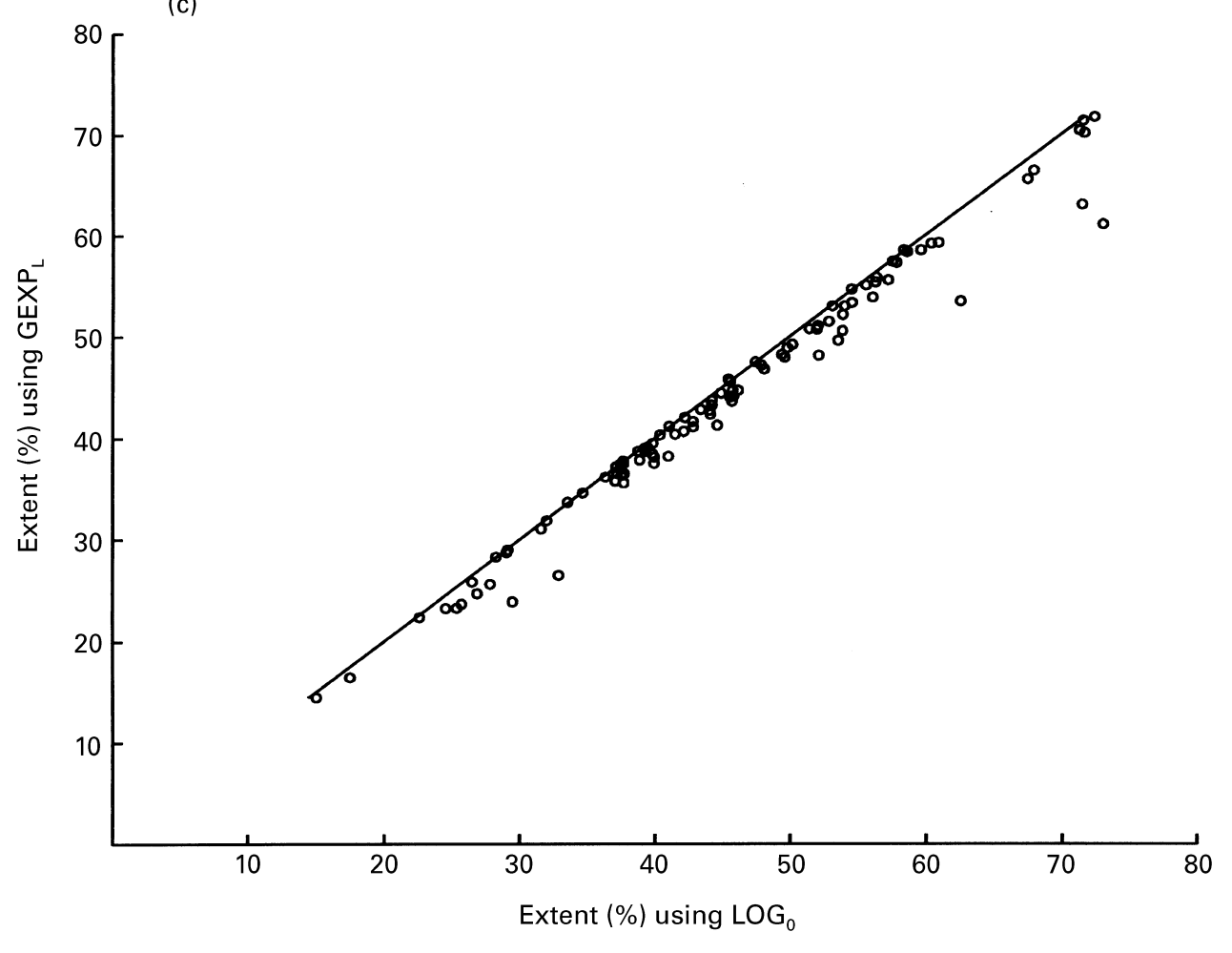

Fig. 3. (Continued)

The models to describe gas production profiles have been extended for multiphasic analysis, assuming that each single phase describes the gas produced by different components of the feedstuff. The LOG and GOMP models have been used multiphasically by Schofield et al. (1994), and the GMM by Cone et al. (1996) and Groot et al. (1996), with two or three phases. This non-linear, multiphasic analysis will increase the number of parameters in the model in comparison with the monophasic analysis, giving rise to a potential decline in robustness of the model (Draper \& Smith, 1981). The models in the present study were derived assuming a single pool of the potentially degradable fraction (France et al. 2000). They may be used additively for multiphasic analysis by assuming that the potentially degradable fraction is composed of more than one pool, and by simply summing over all the pools of degradable material, total gas production according to multiphasic behaviour is obtained. This does not affect the basic assumptions inherent in their derivations.

\section{Yield of gas}

An assumption behind all the gas production equations applied herein is that the rate at which gas is produced is directly proportional to the rate at which substrate is degraded, with constant yield factor Y. Based on stoichiometric relationships, the amount of gas is expected to depend on the relative amounts of VFA formed (Hungate, 1966). The chemical composition of the substrate influences the molar proportions of the major VFA formed (Murphy et al. 1982). Indeed, Beuvink \& Spoelstra (1992) reported a much higher gas yield with rice starch $(0.38 \mathrm{ml} / \mathrm{mg}$ organic matter $(\mathrm{OM}))$ than with glucose $(0.29 \mathrm{ml} / \mathrm{mg} \mathrm{OM})$ or cellulose $(0.30 \mathrm{ml} / \mathrm{mg} \mathrm{OM})$, and rice starch showed significantly higher acetic and butyric acid and a lower propionic acid: total VFA ratio in the vessel. The end-products of fermentation (e.g. VFA and lactic acid) interact with the buffer solution in the medium (Beuvink \& Spoelstra, 1992; Blümmel \& Ørskov, 1993). Beuvink \& Spoelstra (1992) reported that each mol of VFA produced released $0.87 \mathrm{~mol}$ gas from the buffer. Blümmel \& Ørskov (1993) found that on average $50 \%$ of the gas volume consisted of $\mathrm{CO}_{2}$ and $\mathrm{CH}_{4}$ arising from fermentation of substrate, the remainder being $\mathrm{CO}_{2}$ released from the buffer. Cone et al. (1996) reported that incubation of higher amounts of maize cob mix DM (between 0.5 and $1.5 \mathrm{~g} \mathrm{DM} / 100 \mathrm{ml}$ medium) resulted in lower amounts of gas formed per unit DM incubated (between 0.30 and $0.35 \mathrm{ml} / \mathrm{mg} \mathrm{DM}$ ), but Theodorou et al. (1994) observed a constant gas yield of $0.31 \mathrm{ml} / \mathrm{g}$ ryegrass DM degraded, independent of amount of substrate incubated (between 0.2 and $2.0 \mathrm{~g} \mathrm{DM} / 100 \mathrm{ml}$ medium). Pell \& Schofield (1993) and Schofield et al. (1994) stated that gas production was relatively constant when incubated substrates were roughages $(0 \cdot 35-0.39 \mathrm{ml} / \mathrm{mg}$ neutral-detergent fibre degraded). However, close inspection of the graph of gas production against amount of neutral-detergent fibre degraded reported by Pell \& Schofield (1993) shows consistent deviations from the regression line (observed gas production higher than predicted when amount of fibre incubated was low, and lower than predicted when amount of fibre was high). Finally, a variable part of the substrate degraded will not be fermented to VFA and gases, 
but rather incorporated directly into microbial biomass, giving rise to a negative correlation between the amount of gas produced and the microbial biomass yield per unit truly degraded substrate (Blümmel et al. 1997). Thus, there are indications that the gas yield (in units of gas produced per unit substrate truly degraded) is not necessarily constant during the course of incubation.

Possible differences in gas yield per unit of substrate degraded are not directly important in the calculation of extent of ruminal degradation, as can be seen from the absence of $\mathrm{Y}$ in the equations to determine this extent (France et al. 2000). However, if $\mathrm{Y}$ varies during the course of incubation, then the rate of gas production does not properly reflect the rate of substrate degradation. For example, a low yield at the start and a high yield towards the end of the incubation period will underestimate the rate of substrate degradation and consequently the extent of degradation. In particular, substrates with different chemical entities (e.g. fibre, starch, sugars) will be affected because starch and sugars generally have a higher fractional degradation rate than fibre and cause a lower $\mathrm{pH}$ in the rumen fluid (Tamminga et al. 1990) and the VFA molar proportions of each entity and between roughages and concentrates will differ (Murphy et al. 1982). Recent results of a simulation study using the $\mathrm{GEXP}_{\mathrm{L}}$ model indicated that the predicted extent of degradation is closer to the true extent of degradation, if the gas production measured at various time points during incubation is corrected based on the VFA profile at those time points (Dijkstra et al. 1999). Feedstuffs comprising a single entity (e.g. fibre) are probably less prone to differences in VFA molar proportions. If a lack of essential growth factors occurs during the course of incubation, the substrate utilization efficiency for microbial growth will decrease (Pirt, 1975). This will affect the partitioning of degraded substrate between direct incorporation and fermentation, giving rise to an increase in the amount of gas formed per unit substrate degraded. Thus, for various cereal straws, Blümmel \& Ørskov (1993) reported an increased gas yield when incubation time was longer (from an average 0.33 to $0.40 \mathrm{ml} / \mathrm{mg}$ OM degraded at 24 and $48 \mathrm{~h}$ incubation respectively). Therefore, a caveat when applying the gas production equations derived in the present study is, whilst gas yield per unit of substrate degraded (Y) does not directly appear in the calculation of extent of ruminal degradation $(\mathrm{E})$, differences in $\mathrm{Y}$ during the course of fermentation might well affect the value of $\mathrm{E}$.

\section{Microbial protein supply}

Microbial protein supply to the duodenum is an important characteristic in the evaluation of feedstuffs for animal production. In current protein evaluation systems for ruminants, microbial protein supply is predicted using equations which relate microbial protein synthesis (g microbial protein) to the amounts of ruminally degradable OM (e.g. Agricultural and Food Research Council, 1992; Tamminga et al. 1994). Microbial protein synthesis in the rumen may be estimated from the gas production technique as:

$$
\text { microbial protein synthesis }=Y_{M} \times \mathrm{E} \times \mathrm{S}_{0} \text {, }
$$

where $Y_{M}$ is the yield of microbial protein synthesized (g microbial protein/g OM degraded) and $\mathrm{E}$ and $\mathrm{S}_{0}$ are as previously defined but pertain to OM. $Y_{M}$ has been assigned the value of $0.15 \mathrm{~g} / \mathrm{g}$ in the Dutch protein evaluation system (Tamminga et al. 1994), though other values could also be adopted. The microbial protein synthesis equation cannot be applied to feedstuffs with a high protein content. The energy derived by rumen micro-organisms from fermentation of protein is low compared with that from carbohydrates, and would not sustain microbial growth (Russell et al. 1983). $Y_{M}$ has been shown to vary with differences in feed intake level and feedstuffs. Its application in protein evaluation systems is discussed by Dijkstra et al. (1998).

\section{Substrate evaluation}

Bearing in mind the limitations discussed earlier, in vitro gas production is becoming ever more popular in substrate evaluation, particularly for screening purposes. Tamminga $\&$ Williams (1998) recently reviewed the role of the gas production technique to predict nutrient supply in ruminants. The popularity of in vitro gas production stems mainly from (1) the ability to exercise experimental control, (2) the capacity to screen a large number of substrates, (3) the kinetic information which may be derived which, although possible, is a limitation of the end-point techniques such as in vitro digestibility (Tilley \& Terry, 1963) and (4) the fact that no assumptions need be made on the degradability of the soluble fraction of the substrate, as is required for the in sacco technique (Ørskov \& McDonald, 1979). Potential use of gas production to approximate in vivo kinetics is a major expectation and some studies have been published (Blümmel \& Ørskov, 1993; Khazaal et al. 1993, 1994; Sileshi et al. 1996; Lopez et al. 1998), where it has been compared with the in situ polyester bag method. The models discussed in the present paper should help to interpret gas production profiles and provide estimates of kinetic parameters and other biological quantities, including the extent of degradation. In our derivation of the models, a link between the gas production profiles and the extent of degradation in the rumen proper was established (France et al. 2000). This requires knowledge of the potentially degradable and undegradable fractions of the substrate. The amount of substrate at the end of incubation, when fermentation ceased, is the apparently undegradable residue. The DM of this residue may be used to calculate in vitro digestibility as an approximation for the method of Tilley \& Terry (1963) and other methods. Substrate residue remaining after completion of fermentation correlates well with in vitro digestibility (Sileshi, 1995). Further chemical analysis of this residue can provide more information on the individual fractions that constitute the DM, including microbial contamination. To avoid underestimating bias, some allowance may have to be made for the soluble but undegradable fraction which may not be recovered during filtering. In the present analysis, the estimated extent of degradation obtained for each profile showed consistency between the models apart from a mean bias, and consequently the choice of model will not affect the ranking of individual substrates. Finally, using a different fractional passage rate constant $(0.02 / \mathrm{h}$ in the present analysis, indicative of maintenance 
level of feed intake) does change the absolute differences between extent of degradation predicted by the models, but does not affect the ranking between the models (results not shown). The fractional passage rate of particles may be correlated to the incubation time and the fractional rate of degradation. The reason is that the functional density of particles varies with incubation time and degradation rate (Nocek \& Kohn, 1987) and the escape of particles from the rumen depends on its functional density (Kaske \& von Engelhardt, 1990). However, similar generalizations for the fractional passage rate are beyond the scope of this paper, as the objective is confined to the interpretation of gas production curves.

\section{Conclusions}

Increasing knowledge of chemistry and biology of gas production appears to suggest a role for it in animal science. Nutritional significance of gas production can be enhanced and made clearer through better chemical, biological and mathematical understanding. It may be possible to derive biological quantities important for animal performance from the gas production profiles under controlled conditions, through application of sound models to describe the profiles. These biological quantities include the extent of degradation of substrate and the microbial protein synthesized. As for the quantitative description of gas production profiles, non-sigmoidal functions (MM and EXP) and sigmoidal growth functions (GOMP and LOG) have major limitations. The GEXP and GMM models seem particularly suited because of their flexibility to encompass sigmoidal and non-sigmoidal shapes of gas production profiles, whether symmetrical or not.

\section{Acknowledgements}

Financial support for this project through a NATO Collaborative Research Grant (CRG 960240) is gratefully acknowledged. The research of J. Dijkstra has been made possible by a fellowship from the Royal Netherlands Academy of Arts and Sciences.

\section{References}

Agricultural and Food Research Council (1992) AFRC Technical Committee on responses to nutrients. Report no. 9. Nutritive requirements of ruminant animals: protein. Nutrition Abstracts and Reviews, Series B 62, 787-835.

Beuvink JMW \& Kogut J (1993) Modelling gas production kinetics of grass silages incubated with buffered ruminal fluid. Journal of Animal Science 71, 1041-1046.

Beuvink JMW \& Spoelstra SF (1992) Interactions between substrate, fermentation end-products, buffering systems and gas production upon fermentation of different carbohydrates by mixed rumen microorganisms in vitro. Applied Microbiology and Biotechnology 37, 505-509.

Bibby J \& Toutenberg H (1977) Prediction and Improved Estimation in Linear Models. London: John Wiley \& Sons.

Blümmel M \& Ørskov ER (1993) Comparison of in vitro gas production and nylon-bag degradability of roughage in predicting feed intake in cattle. Animal Feed Science and Technology 40, 109-119.
Blümmel M, Steingaß H \& Becker K (1997) The relationship between in vitro gas production, in vitro microbial biomass yield and ${ }^{15} \mathrm{~N}$ incorporation and its implications for the prediction of voluntary feed intake of roughages. British Journal of Nutrition 77, 911-921.

Cone JW, Van Gelder AH, Visscher GJW \& Oudshoorn L (1996) Influence of rumen fluid and substrate concentration on fermentation kinetics measured with a fully automated time related gas production apparatus. Animal Feed Science and Technology 61, 113-128.

Dijkstra J, France J \& Davies DR (1998) Different mathematical approaches to estimating microbial protein supply in ruminants. Journal of Dairy Science 81, 3370-3384.

Dijkstra J, France J, Lopez S \& Dhanoa MS (1999) Impact of variation in yield of gas during incubation on simulated gas production and extent of ruminal degradation. In Proceedings of the British Society of Animal Science, p. 38. Edinburgh: British Society of Animal Science.

Draper NR \& Smith H (1981) Applied Regression Analysis. New York, NY: John Wiley \& Sons.

France J, Dhanoa MS, Theodorou MK, Lister SJ, Davies DR \& Isac D (1993) A model to interpret gas accumulation profiles associated with in vitro degradation of ruminant feeds. Journal of Theoretical Biology 163, 99-111.

France J, Dijkstra J, Dhanoa MS, Lopez S \& Bannink A (2000) Estimating the extent of degradation of ruminant feeds from a description of their gas production profiles observed in vitro: derivation of models and other mathematical considerations. British Journal of Nutrition 83, 143-150.

France J, Dijkstra J, Lopez S \& Dhanoa MS (1998) Models for interpreting in vitro gas production profiles from ruminant feeds. In In Vitro Techniques for Measuring Nutrient Supply to Ruminants, BSAS Publication no. 22, pp. 79-80 [ER Deaville, E Owen, AT Adesogan, C Rymer, JA Huntington and TLJ Lawrence, editors]. Edinburgh: British Society of Animal Science.

Genstat 5 Committee (1987) Genstat 5 Reference Manual. Oxford: Clarenden Press.

Groot JCJ, Cone JW, Williams BA, Debersaques FMA \& Lantinga EA (1996) Multiphasic analysis of gas production kinetics for in vitro fermentation of ruminant feeds. Animal Feed Science and Technology 64, 77-89.

Hungate RE (1966) The Rumen and its Microbes. New York, NY: Academic Press Inc.

Kaske M \& von Engelhardt W (1990) The effect of size and density on mean retention time of particles in the gastrointestinal tract of sheep. British Journal of Nutrition 63, 457-465.

Khazaal K, Boza J \& Ørskov ER (1994) Assessment of phenolicsrelated antinutritive effects in Mediterranean browse: a comparison between the use of the in vitro gas production technique with or without insoluble polyvinylpolypyrrolidone or nylon bag. Animal Feed Science and Technology 49, 133-149.

Khazaal K, Dentinho MT, Ribeiro JM \& Ørskov ER (1993) A comparison of gas production during incubation with rumen contents in vitro and nylon bag degradability as predictors of the apparent digestibility in vivo and the voluntary intake of hays. Animal Production 57, 105-112.

Lopez S, Carro MD, Gonzalez JS \& Ovejero FJ (1998) Comparison of different in vitro and in situ methods to estimate the extent and rate of degradation of hays in the rumen. Animal Feed Science and Technology 73, 99-113.

Motulsky HJ \& Ransnas LA (1987) Fitting curves to data using nonlinear regression: a practical and nonmathematical review. FASEB Journal 1, 365-374.

Murphy MR, Baldwin RL \& Koong LJ (1982) Estimation of stoichiometric parameters for rumen fermentation of roughage and concentrate diets. Journal of Animal Science 55, 411-421. 
Nocek JE \& Kohn RA (1987) Initial particle form and size on change in functional specific gravity of alfalfa and timothy hay. Journal of Dairy Science 70, 1850-1863.

Ørskov ER \& McDonald I (1979) The estimation of protein degradability in the rumen from incubation measurements weighted according to rates of passage. Journal of Agricultural Science, Cambridge 92, 499-503.

Pell AN \& Schofield P (1993) Computerized monitoring of gas production to measure forage digestion in vitro. Journal of Dairy Science 76, 1063-1073.

Pirt SJ (1975) Principles of Microbe and Cell Cultivation. Oxford: Blackwell Scientific Publications.

Ross GJS (1987) MLP Maximum Likelihood Program, version 3.08. Oxford: Numerical Algorithms Group.

Russell JB, Sniffen CJ \& Van Soest PJ (1983) Effect of carbohydrate limitation on degradation and utilization of casein by mixed rumen bacteria. Journal of Dairy Science $\mathbf{6 6}$, 763-775.

Schofield P, Pitt RE \& Pell AN (1994) Kinetics of fibre digestion from in vitro gas production. Journal of Animal Science $\mathbf{7 2}$, 2980-2991.

Sileshi Z (1995) Development of a simple in vitro gas production technique using a pressure transducer, to estimate rate of digestion of some Ethiopian forages. $\mathrm{PhD}$ Thesis, University of Reading.

Sileshi Z, Owen E, Dhanoa MS \& Theodorou MK (1996) Prediction of in situ rumen dry matter disappearance of Ethiopian forages from an in vitro gas production technique using a pressure transducer, chemical analysis or in vitro digestibility. Animal Feed Science and Technology 61, 73-87.

Tamminga S, Van Straalen WM, Subnel APJ, Meijer RGM, Steg A, Wever CJG \& Blok MC (1994) The Dutch protein evaluation system: the DVB/OEB-system. Livestock Production Science 40, 139-155.
Tamminga S, Van Vuuren AM, Van der Koelen CJ, Ketelaar RS \& Van der Togt PL (1990) Ruminal behaviour of structural carbohydrates, non-structural carbohydrates and crude protein from concentrate ingredients in dairy cows. Netherlands Journal of Agricultural Science 38, 513-526.

Tamminga S \& Williams BA (1998) In vitro techniques as tools to predict nutrient supply in ruminants. In In Vitro Techniques for Measuring Nutrient Supply to Ruminants, BSAS Publication no. 22, pp. 1-11 [ER Deaville, E Owen, AT Adesogan, C Rymer, JA Huntington and TLJ Lawrence, editors]. Edinburgh: British Society of Animal Science.

Theodorou MK, Williams BA, Dhanoa MS, McAllan AB \& France J (1994) A simple gas production method using a pressure transducer to determine the fermentation kinetics of ruminant feedstuffs. Animal Feed Science and Technology 48, 185-197.

Tilley JMA \& Terry RA (1963) A two stage technique for the in vitro digestion of forage crops. Journal of the British Grassland Society 18, 104-111.

Tukey JW (1977) Exploratory Data Analysis. Reading, MA: Addison-Wesley.

Williams BA, Chuzaemi S, Soebarinoto, Van Bruchem J, Boer H \& Tamminga $S$ (1996) A comparison of ten rice-straw varieties grown at two different altitudes during a wet and a dry season, using the in vitro cumulative gas production technique. Animal Feed Science and Technology 57, 183-194.

Williams BA, Van der Poel AFB, Boer H \& Tamminga S (1995) The use of cumulative gas production to determine the effect of steam explosion on the fermentability of two substrates with different cell wall quality. Journal of the Science of Food and Agriculture 69, 33-39.

Zwietering MH, Jongenburger I, Rombouts FM \& Van 't Riet K (1990) Modelling of the bacterial growth curve. Applied and Environmental Microbiology 56, 1875-1881. 